\title{
Trustworthiness Assessment in Mapping Urban Accessibility via Sensing and Crowdsourcing
}

\author{
Catia Prandi \\ Department of \\ Computer Science and Engineering \\ University of Bologna \\ Via Mura Anteo Zamboni 7 \\ 40127 Bologna (BO), Italy \\ $+390547338813$ \\ catia.prandi2@unibo.it
}

\author{
Silvia Mirri \\ Department of \\ Computer Science and Engineering \\ University of Bologna \\ Via Mura Anteo Zamboni 7 \\ 40127 Bologna (BO), Italy \\ $+390547338813$ \\ silvia.mirri@unibo.it
}

\author{
Paola Salomoni \\ Department of \\ Computer Science and Engineering \\ University of Bologna \\ Via Mura Anteo Zamboni 7 \\ 40127 Bologna (BO), Italy \\ +390547338813 \\ paola.salomoni@unibo.it
}

\begin{abstract}
In this work we present the trustworthiness assessment in mPASS (mobile Pervasive Accessibility Social Sensing), a location and context aware system that collects data from crowdsourcing and sensing in order to map urban and architectural accessibility. The fusion of heterogeneous urban sources allows mPASS to provide users with personalized paths, computed on the basis of their preferences and needs. To perform this task, the system needs a set of georeferenced data that is dense enough and trustworthy enough to avoid false positives and negatives, e.g. to prevent users from encounter on their path an unknown barrier or a non-existing facility. In order to reach this goal, we propose a trustworthiness assessment which combines accuracy of sensors, source credibility of the crowd and the authority of experts. To evaluate the effectiveness of our trustworthiness assessment, we conducted a set of simulations by exploiting OSM data and we have obtained interesting results.
\end{abstract}

\section{Categories and Subject Descriptors}

C.2.4 [Computer-Communication Networks]: Distributed Systems - distributed applications. H.1.2 [Models and Principles]: User/Machine Systems - Human information processing.

\section{General Terms}

Experimentation, Human Factors.

\section{Keywords}

Trustworthiness, urban accessibility, crowdsourcing, sensing.

\section{INTRODUCTION}

The availability of information about urban accessibility is very limited if compared with georeferenced data related to other topics. This is fundamentally due to the apparently limited audience of accessibility related information, that are generally considered useless or not of interest for the crowd. Actually, the group of people who can benefit from such a kind of information is widely larger that this: from parents with prams to elderly people, several citizens can take advantage of detailed information

Permission to make digital or hard copies of part or all of this work for personal or classroom use is granted without fee provided that copies are not made or distributed for profit or commercial advantage and that copies bear this notice and the full citation on the first page. Copyrights for third-party components of this work must be honored. For all other uses, contact the Owner/Author.

Copyright is held by the owner/author(s).

Urb-IoT '14, Oct 27-28 2014, Rome, Italy

ACM 978-1-4503-2966-8/14/10.

http://dx.doi.org/10.1145/2666681.2666703 on pathways, ramps, street lights, crossing and bus stops facilities.

Differently from subjective information/review about specific services and places, data about urban accessibility are objectively measurable [1]. When barriers/facilities are evaluated by experts (e.g. people working for authorities and organizations), data about their presence or absence can be considered totally trustworthy. Unfortunately, these evaluations are too few to be significant in effectively computing a personalized route according to specific user's preferences and needs. To overcome this lack, many systems use crowdsourcing to gather information about barriers and facilities [6], involving volunteers who are directly interested in such kind of information and/or who are motivated by their knowledge on local environments. Besides crowdsourced data, some georeferenced applications use sensors embedded in consumer electronics devices (including tablet PCs, smartphones and smart watches). The large availability of such sensor-equipped devices and their accuracy make information gathered from sensing a good opportunity to improve the quantity of data about urban accessibility.

Some issues about the quality of collected information arise due to the accuracy of sensors and the credibility of users involved in the data gathering. Although any instance of the crowdsourced and sensed data may be unreliable, aggregating a large number of information related to a barrier/facility makes the data more trustworthy. The error made by a single sensor or user become less significant while the volume of the data increases.

With the aim of collecting data about urban accessibility and providing users with personalized paths, we have developed mPASS (mobile Pervasive Accessibility Social Sensing) [4]. This poster proposes the mPASS trustworthiness assessment, showing how it combines accuracy of sensors, source credibility of the crowd and the authority of experts

\section{THE MPASS SYSTEM}

In this section we are going to present some main issues related to the mPASS system, which is deeply described in $[4,5]$, while in this paper we are going to focus on trustworthiness assessment.

In order to provide citizens with personalized georeferenced information and routing services related to the urban environment, mPASS works on heterogeneous information, coming from different sources: (i) sensors, (ii) crowdsourcing, (iii) official reviews.

In (i) mobile devices are used as sensors with the aim of producing georeferenced data, while users are moving in the urban environment. While the mPASS app is running on the devices, it can identify simple barriers (e.g. steps) and facilities (e.g. ramps), by exploiting sensors like gyroscope, accelerator and 
GPS, which are usually installed on mobile devices. It is worth noting that multiple sensing of the same barrier/facility can improve the data accuracy, while data sensed by just a single user can be intended as not very accurate.

Data are collected by means of mobile devices also in case of (ii). In particular, mPASS app collects reviews about urban accessibility from users in two different ways:

1. Volunteering: a user who wants to send a review about the place use mPASS to fill a related form.

2. On demand: the mPASS app can ask users to fill a form of a review about a place where they are (or nearby). The system sends a notification to the users with the review request. The notification system is strategic in mPASS, in order to add data about some specific aPOIs.

Accuracy of reviews gathered via crowdsourcing is strictly related to the number of users who reviewed those barrier/facilities and to their credibility.

The (iii) source of data is based on official reviews: experts from authorities and organizations (e.g., local administrations, disability right organizations, hotels associations, etc.) produce reviews about barriers and facilities, rating the accessibility of indoor and outdoor places. Municipalities and other public administrations (such as bus operating companies [7]) offer their open data, which represent an accurate data source for the mPASS system.

All these data collected by mPASS from different sources are organized as reports about aPOIs. In particular, an aPOI can be associated to one or more accessibility reports, which can be categorized according to the data sources: (i) S-report (sensor report); (ii) U-report (user report); (iii) E-report (export report). Obviously, mPASS manages multiple reports for each aPOI, classified with one or more different source classes and combined as described in the following section.

An aPOI is a part of the urban outdoor environment (a urban design aPOI). In particular, we considered barriers/facilities related to: (i) gap; (ii) cross; (iii) obstruction; (iv) parking; (v) surface; (vi) pathway; (vii) light; (viii) bus stop.

Obviously, only a subset of barriers/facilities can be identified by sensing activity. Examples of aPOIs that can be sensed are steps and stairs (detectable by a single walking pedestrian) or ramps and curb (detectable by wheelchair users).

\section{TRUSTWORTHINESS ASSESSMENT 3.1 Unreliable Data}

The presence or the absence of an aPOI (as a barrier or a facility) can be evaluated by one or more sources and the system has to cope with the availability of multiple data, produced by sources with different levels of trustworthiness. In particular, starting from the whole set of reports related to a specific aPOI, mPASS has to decide if there is a barrier or a facility, what type it is and which characteristics it has got. This information is needed both to compute a route and to show an accessibility map of a specific area. While deciding, on the basis on possible unreliable reports, mPASS can generate different types of error:

1. Undetected existing barrier: mPASS could produce paths or maps without considering the presence of an existing barrier.

2. Undetected exiting facility: mPASS could suggest paths without taking into account an undetected facility.

3. Detected non-existing barrier: mPASS could avoid an accessible path due to the incorrect detection of a non-existing barrier.
4. Detected non-existing facility: mPASS could suggest a route considering it as adequate because of the incorrect detection of a non-existing facility.

It is worth noting that all the four types of error can cause difficulties to users, but cases 1 and 4 are more critical than the other ones because they could prevent the user from reaching his/her goal or destination.

\subsection{Report Trustworthiness}

In order to avoid problems related to unreliable, mPASS models and manages trustworthiness of data, starting from report trustworthiness. This is a continuous value ranging from 0 to 1 , where 0 means that the report is totally unreliable, while 1 means the report is totally trustworthy.

We consider the report trustworthiness as a result of the type of source and its quality in terms of reliability.

It is worth noting that these sources are involved in stating the presence or the absence of a barrier/facility in the urban environment. This means that they are not engaged in rating the quality of an aPOI, by expressing a value in a prefixed range, like in more common rating systems. Hence, in order to map the presence/absence of an aPOI, we have studied a model which takes into account different characteristics of our data sources and which aims to merge them together. To reach this goal, trustworthiness of these kinds of sources is assessed in three different ways, as follows.

Experts are totally trustworthy by definition, since they are professionally working in order to do an accessibility census and they have the knowledge, the expertise and the tools needed to do assess the presence of each type of barriers and facilities [2]. Trustworthiness of experts is set as 1 in any E-report: $\mathrm{T}\left(\mathrm{r}_{\mathrm{e}}\right)=1$

The presence of an E-report overcomes all other reports and automatically states the aPOI real condition. aPOIs reported by an expert are used to evaluate trustworthiness of other data sources.

Trustworthiness of a U-report is defined on the basis of user's credibility [3]. Users can do very accurate and reliable evaluations about the presence or the absence of an aPOI, but they can also do (intentionally or not) a wrong one. When a user joins the mPASS system his/her credibility is set to 0.5 ; this means that the user is initially considered as neutral, neither totally credible (credibility set to 1 ), nor totally unreliable (credibility set to 0 ). In order to state his/her credibility to a more realistic value, we compare his/her evaluations of aPOIs with experts' ones. The more they match, and the more the user's credibility grows; if they don't match, the user credibility decreases (ranging in the interval $[0$, 1]). This process is periodically iterated in order to better assess the user's credibility and to follow his/her evolution. Every time a user does a report, the report trustworthiness is set according to his/her credibility (defined as $\mathrm{C}(\mathrm{u})$ ): $\mathrm{T}\left(\mathrm{r}_{\mathrm{u}}\right)=\mathrm{C}(\mathrm{u})$.

Finally trustworthiness of a S-report is computed on the basis of its accuracy. We have to consider that, in this context, sensors don't have to measure a value in a continuous range, but they have to state the presence/absence of a condition.

Sensors accuracy depends from many factors, first of all barriers to sense: for instance, sensing single steps is more difficult than sensing a stair. Other aspects that strongly influence the sensor accuracy are the device in use and the user (i.e. the way he/she walks or runs, his/her speed, etc.). Differently from the user's credibility, we don't change the sensor accuracy value until the user or the device changes. Every time a sensor adds a S-report to 
the mPASS system, its trustworthiness is set to the accuracy of the sensor (defined as $\mathrm{A}(\mathrm{s}))$ : $\mathrm{T}\left(\mathrm{r}_{\mathrm{s}}\right)=\mathrm{A}(\mathrm{s})$

\section{3 aPOI Trustworthiness}

Trustworthiness of an aPOI is computed as follows, considering all the reports available for that aPOI:

- If there is an E-report that states the presence of the barrier/facility, trustworthiness of the aPOI is 1 .

- If there is an E-report that states the absence of the barrier/facility, trustworthiness of the aPOI is -1 .

- In all the other cases, trustworthiness of the aPOI is the average value of all the reports trustworthiness, considering them as positive if they state the presence of the barrier/facility or as negative if they state the absence of the barrier/facility. So the trustworthiness of the aPOI is:

$$
\mathrm{T}(\mathrm{aPOI})=\frac{\left(\sum T\left(r_{s+}\right)+\sum T\left(r_{u+}\right)\right)-\left(\sum T\left(r_{s-}\right)+\sum T\left(r_{u-}\right)\right)}{\# r}
$$

Where $\mathrm{H} r$ is the number of reports available for this aPOI.

It is worth noting that the positive component of the expression $\left(\sum T\left(r_{s_{+}}\right)+\sum T\left(r_{u+}\right)\right)$ sums all the trustworthiness related to U-reports and s-reports that evaluate the detected barrier/facility as present. In the same way, the negative component of the expression $\left(\sum T\left(r_{s_{-}}\right)+\sum T\left(r_{u_{-}}\right)\right)$sums all the trustworthiness related to U-reports and S-reports that evaluate the barrier/facility as absent. As a consequence of this definition, the aPOI trustworthiness ranges from -1 to 1 in the following way:

- the value 1 states the presence of the barrier/facility related to that aPOI; this value can't vary if it is due to an E-report.

- All the positive values represent different grades of trustworthiness about the presence of the barrier/facility.

- The value 0 represents the total uncertainty about the presence or absence of the barrier/facility. There are no positive or negative clues to decide if the barrier/facility is there or not.

- All the negative values represent different grades of trustworthiness about the presence of the barrier/facility.

- The value -1 states the absence of the barrier/facility related to that aPOI; this value can't vary if it is due to an E-report.

To avoid false positives and false negatives, mPASS needs to remove uncertainty about a barrier/facility as fast as it possible, e.g. obtaining more reports for those aPOIs which trustworthy value is near to 0 . With this aim, when an aPOI trustworthiness ranges in the interval $[-0.5,+0.5]$, mPASS starts the notification mechanism and sends a report request to each user with a high credibility, who is in proximity of the aPOI. In order to collect reports with a high value of trustworthiness, the system selects more credible users, so as to better assess the presence/absence of the related barrier/facility. Experts can be involved by the notification system, since they can definitively state the presence/absence of the barrier/facility. The aPOI trustworthiness is periodically re-computed, so as to take into account consolidated values related to $\mathrm{A}(\mathrm{s})$ and updated values related to $\mathrm{C}(\mathrm{u})$, which can change over time.

\section{SIMULATIONS}

With the aim of conduct a first evaluation of our trustworthiness assessment system we have simulated it on a discrete-event multiagent simulation library developed in Java called MASON (http://cs.gmu.edu/ eclab/projects/mason/). In order to simulate our model in a real urban scenario, we used GeoMason, a MASON extension that adds support for vector and raster geospatial data. In particular, the aim of such simulations is to evaluate how many reports have to be collected (in average conditions), so as to let the system works with stable and trustworthy data. To reproduce the urban environment, we have imported data provided by OpenStreetMap (OSM). In particular, we have exported the OSM data of a specific area that represents the center of Cesena (Italy). In this area, experts and volunteers involved in disability organizations have manually reported urban accessibility barriers. We have added such georeferenced data in our simulated environment. To test our trustworthiness assessment system, we limited the aPOI types to stairs and steps, with the aim of verifying the system in a scenario with several and significant S-reports. In the simulated environment, pedestrian agents move in lines that represent streets in Cesena.

The aim of simulations is to monitor trustworthiness assessment system and to verify the system behavior in detecting existing/ non-existing barriers. We have observed that our system allows to minimize the number of unreliable reports, identifying the presence/absence of barriers with a high level of confidence.

\section{REFERENCES}

[1] Carvalho, A., Heitor, T., and Cabrita Reis, A. 2012. Ageing cities: shifting from special needs to inclusive design in urban space. In Proceedings of the $6^{\text {th }}$ European Symposium on Research in Architecture and Urban Design (Porto, Portugal, September 12-15, 2012). EURAU'12.

[2] Flanagin, A. J. and Mertzger, M. J. 2013. Trusting ExpertVersus User-Generated Ratings Online: The Role of Information Volume, Valence and Consumer Characteristics. In Computers in Human Behavior. 29, 4 (July 2013), 16261634. DOI= http://dx.doi.org/10.1016/j.chb.2013.02.001.

[3] Metzger, M. J. and Flanagin, A. J. 2013. Credibility and Trust of Information in Online Environments: The Use of Cognitive Heuristics. In Journal of Pragmatics. 59 (Dec. 2013), 210-220. DOI=http://dx.doi.org/10.1016/j.pragma.2013.07.12.

[4] Mirri, S., Prandi, C., and Salomoni, P. 2014. A Context-Aware System for Personalized and Accessible Pedestrian Paths. In Proceedings of the International Conference on High Performance Computing \& Simulation (Bologna, Italy, July 21-25, 2014). HPCS' 2014.

[5] Prandi, C., Salomoni, P. and Mirri, S. 2014. mPASS: Integrating People Sensing and Crowdsourcing to Map Urban Accessibility. In Proceedings of the IEEE International Conference on Consumer Communications and Networking Conference (Las Vegas, Nevada, USA, January 10-13, 2014). CCNC'14.

[6] Song, W. and Sun, G. 2010. The Role of Mobile Volunteered Geographic Information in Urban Management. In Proceeding of the $18^{\text {th }}$ International Conference on Geoinformatics (Beijing, China, June 18-20, 2010). 1-5. DOI=http://dx.doi.org/10.1109/GEOINFORMATICS.2010.5 567728.

[7] Venanzi, M., Rogers, A., and Jennings, N. R. 2013. Trust-based Fusion of Untrustworthy Information in Crowdsourcing Applications. In Proceedings of the $12^{\text {th }}$ International Conference on Autonomous Agents and Multiagent Systems (Saint Paul, MN, USA, May 06-10, 2013). AAMAS 2013. 829-836. 